\title{
CONCENTRACIÓN DE NITRATOS EN DOS TIPOS COMERCIALES DE LECHUGA (Lactuca sativa L.) CULTIVADAS CON DISTINTAS FUENTES NITROGENADAS
}

\author{
NITRATE CONCENTRATION IN TWO COMMERCIAL TYPES OF \\ LETTUCE (Lactuca sativa L.) GROWN WITH DIFFERENT NITROGEN \\ SOURCES
}

\author{
Ricardo Tomás Ollúa ${ }^{1}$, Verónica Romina Logegaray ${ }^{1}$, y Ángel Chiesa ${ }^{1 *}$ \\ ${ }^{1}$ Facultad de Agronomía, Universidad de Buenos Aires, Av. San Martín 4453 C1417DSE. CABA, \\ Argentina. \\ *Autor para correspondencia E mail: achiesa@agro.uba.ar
}

\section{RESUMEN}

Los nitratos $\left(\mathrm{NO}_{3}^{-}\right)$ingeridos por el hombre pueden convertirse a nitritos $\left(\mathrm{NO}_{2}^{-}\right)$formando metahemoglobina y nitrosaminas carcinogénicas. La lechuga se encuentra entre las hortalizas con mayor capacidad de acumulación de nitratos. El objetivo del estudio fue evaluar la respuesta del cultivo de lechuga a dosis crecientes de nitrógeno aplicado como urea y al agregado de lombricompuesto, sobre la concentración de nitratos en dos tipos comerciales (hoja suelta y mantecosa). En un ensayo se incorporó abono orgánico $24 \mathrm{t} \mathrm{ha}^{-1}$ de lombricompuesto (L1) y se comparó un testigo sin abonar (L0). En simultaneo se realizó un ensayo que comparó un testigo sin fertilizar (N0) con $75 \mathrm{~kg} \mathrm{~N} \mathrm{ha}^{-1}$ (N1) y $150 \mathrm{~kg} \mathrm{~N} \mathrm{ha}^{-1}(\mathrm{~N} 2)$ aplicado como urea. Estos ensayos se realizaron en dos épocas de producción: otoño-invierno y primavera. En los experimentos, el diseño experimental fue en bloques completos al azar con parcelas divididas y con 4 repeticiones. Durante el cultivo y a la cosecha se determinó la concentración de $\mathrm{NO}_{3}^{-}$por colorimetría. Se observó un nivel decreciente de nitratos para ambos ensayos durante el período invernal. El genotipo influyó en la concentración de nitratos, según tratamientos y épocas. Para el tratamiento con lombricompuesto se evidenció una mayor proporción de nitratos para 'Brisa' (hoja suelta) en ambas épocas. En otoño-invierno, 'Brisa' reflejó respuesta a la fertilización con urea mostrando mayores niveles de nitratos a cosecha con respecto a 'Daguan'. En primavera no hubo respuesta a la fertilización y solo el tratamiento con $150 \mathrm{~kg} \mathrm{~N} \mathrm{ha}^{-1}$ (N2) en 'Brisa' presentó mayores niveles de nitratos en hoja a cosecha.

Palabras clave: fertilización química, urea, lombricompuesto, compuestos carcinogénicos, nitritos.

\section{ABSTRACT}

Nitrate $\left(\mathrm{NO}_{3}^{-}\right)$can transform into nitrite $\left(\mathrm{NO}_{2}^{-}\right)$in the human body and nitrites can change normal hemoglobin to methemoglobin, contributing to the formation of carcinogenic nitrosamines. Lettuce is one of the vegetables with a high capacity to accumulate nitrates. The objective of this study was to evaluate the response of lettuce to increasing doses of nitrogen applied as urea and the addition of vermicompost on the nitrate leaf content of two commercial types of lettuce: cv. Daguan (butterhead) and cv. Brisa (loose-leaf). Two field trials were conducted simultaneously in two growing seasons: autumn-winter and spring. One trial consisted of compost $24 \mathrm{tha}^{-1}$ vermicompost-(L1) added and a control treatment (L0) with no amendment, while the other treatment consisted of $75 \mathrm{~kg} \mathrm{~N} \mathrm{ha}^{-1}(\mathrm{~N} 1)$ 
and $150 \mathrm{~kg} \mathrm{~N} \mathrm{ha}^{-1}$ (N2) applied as urea, also with a control treatment without fertilization (N0). The experimental design was a randomized complete block, split-plot design with four replications. Nitrate concentration was measured by colorimetry during growth and harvest. A decreasing level of nitrate concentration was observed in both trials during the winter period. Nitrate concentration was influenced by genotype, fertilization treatments and seasons. A higher level of nitrates was observed in the vermicompost treatment with 'Brisa' in both seasons. In the autumn-winter season, 'Brisa' presented a higher response to urea application, recording higher levels of nitrates at harvest compared to 'Daguan'. No response to fertilization was observed in the spring and only the treatment consisting of $150 \mathrm{~kg} \mathrm{~N} \mathrm{ha}^{-1}$ (N2) in 'Brisa' resulted in higher leaf nitrate content at harvest.

Key words: chemical fertilizer, urea, compost, carcinogenic compounds, nitrites

\section{INTRODUCCIÓN}

La lechuga puede cultivarse en una amplia gama de suelos, aunque su mejor desarrollo se logra en suelos arcillo-arenosos con adecuado tenor de materia orgánica. Se adapta bien a suelos alcalinos y tolera niveles medios de salinidad del suelo; esto último es muy variable según cultivar (de Grazia et al, 2001). Para la mayoría de los cultivos, el nitrógeno es el principal factor limitante de los rendimientos (Chowdhury y Das, 2015), el cuál es absorbido mayormente como nitratos o amonio. El nitrógeno absorbido representa entre 1,5 a 5\% del peso fresco. El 50\% forma parte de compuestos de elevado peso molecular como proteínas y ácidos nucleicos, mientras que el otro $50 \%$ se presenta como compuestos inorgánicos, nitratos y amonio (Azcon-Bieto y Talon, 2000). Para lograr buenos rendimientos comerciales la lechuga requiere entre 90 a $200 \mathrm{~kg}$ de $\mathrm{N} \mathrm{ha}^{-1}$ dependiendo del contenido de agua del suelo, relacionado con efecto osmótico de los nitratos (Tei et al, 2003).

La lechuga es uno de los vegetales que tiende a acumular mayor cantidad de nitratos (Bahadoran et al., 2016; Hord et al., 2009). El manejo del cultivo influye en la acumulación de nitratos (Harrison, 2001, Konstantopoulou et al., 2010). Se han observado variaciones en la cantidad de nitratos según la época de producción (Escobar-Gutierrez, 2002; Pavlou et al, 2007; Koudela y Petøíková. 2008; Konstantopoulou et al, 2010), la tecnología empleada ya sea con el uso de fertilizantes de síntesis química o de enmiendas orgánicas (De Martin y Restani, 2003; Escalona et al, 2009) y el genotipo (Siomos, 2002; Chiesa, 2010). La acumulación de nitratos es el resultado de un desbalance entre suministro o disponibilidad de nitrógeno y la demanda para crecimiento del cultivo (Burns et al., 2004). Esto se da especialmente en invierno cuando el crecimiento disminuye proporcionalmente más que la absorción de nitrógeno y además la radiación solar es menor, la cual influye sobre la actividad de la nitrato reductasa.

Los productos frutihortícolas con elevados contenidos de nitratos pueden ocasionar daños indirectos en la salud de los consumidores. En los últimos años se han intensificado las investigaciones orientadas a conocer las características higiénico-sanitarias en frutas y hortalizas, para garantizar al consumidor la provisión de productos inocuos. Por tal, la importancia de los nitratos en la salud del hombre deriva del hecho que los nitratos pueden convertirse "in vivo" a nitritos produciendo efectos tóxicos. Los nitritos pueden reaccionar con aminas secundarias o terciarias en el organismo formando compuestos N-nitroso, de efectos cancerígenos (Albornoz, 2000; Filippini, 2006).

Para garantizar la inocuidad de los productos frutihortícolas se establecieron niveles máximos tolerables de nitratos. El Codex Alimentarius establece un rango de ingesta diaria admitida (IDA) de nitratos de 0 a 3,7 $\mathrm{mg} \mathrm{kg}^{-1}$ de peso vivo (WHO, 1995). Para el caso específico de la lechuga, la Comunidad Europea (2006) establece límites máximos de contenido de nitratos en hoja para la lechuga cultivada a campo e invernadero, según época de producción y tipo comercial:

- Lechuga tipo iceberg: $2500 \mathrm{mg} \mathrm{NO}_{3}^{-} \mathrm{kg}^{-1}$ en invernadero; $2000 \mathrm{mg} \mathrm{NO}_{3}^{-} \mathrm{kg}^{-1}$ al aire libre.

- Lechuga fresca (excepto iceberg): invierno: $4500 \mathrm{mg} \mathrm{NO}_{3}^{-} \mathrm{kg}^{-1}$ en invernadero; $4000 \mathrm{mg} \mathrm{NO}_{3}^{-}$ $\mathrm{kg}^{-1}$ aire libre; primavera-verano: $3500 \mathrm{mg} \mathrm{NO}_{3}^{-}$ $\mathrm{kg}^{-1}$ en invernadero, $2500 \mathrm{mg} \mathrm{NO}_{3}^{-} \mathrm{kg}^{-1}$ aire libre.

En Argentina, los alimentos que superen los $200 \mathrm{mg}$ de $\mathrm{NO}_{3}^{-} \mathrm{kg}^{-1}$ de producto deben identificarse con una leyenda de producto no apropiado para consumo a menores de un año (Secretaría de Agricultura, Ganadería, Pesca y Alimentos de la República Argentina, Resolución 256/2007) sin especificar valores por cultivo.

El consumo de lechuga está ampliamente generalizado en Argentina y se estima 20,7 kg por habitante año ${ }^{-1}$, ubicándose en tercer lugar dentro de las hortalizas. La superficie cultivada en el país se estima en 40.000 hectáreas, en especial en los cinturones hortícolas que rodean las grandes ciudades: Buenos Aires, Mar del Plata, Rosario y Santa Fe, Santiago del Estero y Mendoza (Fernández Lozano, 2012). Debido a su importancia en la 
dieta, el efecto del consumo de nitratos sobre la salud y la incidencia de las prácticas de manejo sobre dicho contenido es necesario estudiar el contenido de nitratos en los cultivares más utilizados, la variación en el año y el efecto de factores como la fertilización. El objetivo de este estudio fue evaluar la influencia del genotipo, la aplicación de diferentes fuentes de nitrógeno y la época de producción en la concentración de nitratos en hojas de lechuga.

\section{MATERIALES Y MÉTODOS}

Los ensayos se llevaron a cabo en el campo experimental de la Cátedra de Horticultura de la Facultad de Agronomía de la Universidad de

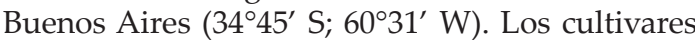
de lechuga (Lactuca sativa L.) utilizados correspondieron a los tipos comerciales hojas sueltas 'Brisa', y mantecosa 'Daguan'.

El cultivo se inició a partir de plantines producidos en invernadero comercial. El transplante se realizó al estado de dos hojas verdaderas totalmente expandidas. Se dispusieron sobre canteros (cuadros) de 1,1 $\mathrm{m}$ de ancho y 0,2-0,3 $\mathrm{m}$ de alto, a una densidad de 16 plantas $\mathrm{m}^{-2}(0,25 \mathrm{~m} \times 0,25 \mathrm{~m})$ para ambos cultivares. El manejo del cultivo fue similar a uno comercial.

El suelo presenta textura franco limosa, con $27,5 \%$ de arcilla, $52,5 \%$ de limo y $20 \%$ de arena, y posee una moderada a baja conductividad hidráulica (28 $\left.\mathrm{mm} \mathrm{hora}^{-1}\right)$ (Tabla 1).

Se realizaron simultáneamente dos ensayos. En el primer ensayo se incorporó abono orgánico de forma manual en el hoyo de plantación a razón de $150 \mathrm{~g}$ de lombricompuesto (L1), (equivale a 24 t ha ${ }^{-1}$ de lombricompuesto) y se comparó con un testigo sin abonar (L0). En la Tabla 1 se presentan las características físico-químicas del lombricompuesto. En el segundo ensayo se aplicó urea al voleo cuando el cultivo cubrió el $60 \%$ de los entresurcos. Se comparó un testigo sin fertilizar (N0) con dos niveles de fertilización: $75 \mathrm{~kg} \mathrm{~N}^{-1}$ (N1) y $150 \mathrm{~kg} \mathrm{~N} \mathrm{ha}^{-1}$ (N2). Ambos ensayos se realizaron en dos épocas de producción (otoño y primavera).

\section{Preparación del extracto para determinar nitratos}

Se utilizó la técnica de Cataldo et al. (1975) ajustada para determinar nitratos en planta. Una vez tomadas las muestras, el material se secó en estufa a $60^{\circ} \mathrm{C}$ con aire forzado hasta peso constante y luego se molió con molinillo. En cada determinación se utilizó $0,5 \mathrm{~g}$ de material molido proveniente de hojas situadas en posición media en la planta, y se colocó en $50 \mathrm{~mL}$ de agua destilada, se hirvió suavemente durante $30 \mathrm{~min}$ en un Erlenmeyer de $250 \mathrm{~mL}$. Durante la ebullición se debió agregar frecuentemente agua destilada para evitar reducción de volumen. Se filtró la muestra y se transfirió cuantitativamente a un matraz de $50 \mathrm{~mL}$, lavando con agua. Se llevó a volumen con agua destilada, se tapó y agitó. La muestra se conservó en heladera a $4^{\circ} \mathrm{C}$.

\section{Determinación colorimétrica}

Se tomaron $0,2 \mathrm{~mL}$ del extracto por duplicado en dos tubos de vidrio de $25 \mathrm{~mL}$, a temperatura ambiente de $20^{\circ} \mathrm{C} \pm 2^{\circ} \mathrm{C}$. Uno de los tubos fue el testigo o blanco y el otro el tubo incógnito. Se agregaron $0,8 \mathrm{~mL}$ de una solución de ácido salicílico al $5 \% \mathrm{p} / \mathrm{v}$ en $\mathrm{H}_{2} \mathrm{SO}_{4}$ al tubo incógnita y 0,8 $\mathrm{mL}$ de ácido $\mathrm{H}_{2} \mathrm{SO}_{4}$ sin el ácido salicílico al tubo testigo. La solución con ácido salicílico se elaboró en el momento de usar. Luego en un agitador se homogeneizó la mezcla en los tubos inmediatamente después de agregar los ácidos, especialmente aquel con ácido salicílico. Luego de $20 \mathrm{~min}$ se agregaron lentamente $19 \mathrm{~mL}$ de $\mathrm{NaOH} 2 \mathrm{~N}$ con dosificador o con una bureta automática, y se homogeneizó con agitador. Se dejó enfriar a temperatura ambiente durante 12 a 24 horas. El color es estable por 48 horas. La lectura en el espectrofotómetro (Agilent 8453, Alemania) se realizó a 410 nm. Para cada muestra la lectura se leyó con su

Tabla 1. Características químicas del suelo de textura franco limosa y del lombricompuesto utilizados en el estudio de nitratos en lechuga.

Table 1. Chemical properties of the silty loam soil and of the vermicompost used in the study of nitrates in lettuce.

\begin{tabular}{|c|c|c|c|c|c|c|c|c|c|c|}
\hline & $\mathrm{pH}$ & $\mathrm{CE}$ & P disponible & C orgánico & $\mathrm{N}$ total & $\mathrm{Ca}^{++}$ & $\mathrm{Mg}^{++}$ & $\mathrm{Na}^{+}$ & $\mathrm{K}^{+}$ & CIC \\
\hline \multirow{3}{*}{ Suelo } & --- & $\mathrm{dS} \mathrm{m}^{-1}$ & $\mathrm{mg} \mathrm{kg}^{-1}$ & $\%$ & $\%$ & $\mathrm{cmol} \mathrm{kg}^{-1}$ & $\mathrm{~mol} \mathrm{~kg}^{-1}$ & $\mathrm{cmol} \mathrm{kg}^{-1}$ & $\mathrm{cmol} \mathrm{kg}^{-1}$ & $\mathrm{cmol} \mathrm{kg}^{-1}$ \\
\hline & & & & & & & & & & \\
\hline & 6,9 & 0,5 & 122 & 2,1 & 0,26 & 13,0 & 2,3 & 0,5 & 1,6 & 21,0 \\
\hline \multicolumn{11}{|c|}{ Lombricompuesto } \\
\hline & 7,34 & 3,38 & 269,9 & 14,6 & 0,91 & 2810,1 & 395,3 & 310,3 & 733,8 & - \\
\hline
\end{tabular}

Fuente: Laboratorio de Análisis de Suelos, Aguas y Vegetales de la Facultad de Agronomía (UBA). 
blanco preparado sin el ácido salicílico.

\section{Cálculos}

Se realizó la curva de calibración con concentraciones conocidas de nitratos y el cero (agua destilada sola). Estas soluciones estándar se prepararon a partir de una solución madre de $\mathrm{NO}_{3} \mathrm{~K}$ $\left(7,22 \mathrm{~g} \mathrm{~L}^{-1}\right)$ que equivale a $1000 \mathrm{cmol} \mathrm{kg}^{-1}$. Se tomaron $10 \mathrm{~mL}$ de la solución madre y se llevaron a matraz de $100 \mathrm{~mL}\left(100 \mathrm{cmol} \mathrm{kg}^{-1}\right)$.

\section{Diseño y análisis estadístico}

Los ensayos de agregado de lombricompuesto (L1) y de urea en dos niveles (N1 y N2) se realizaron en simultáneo comparados con un control, en dos épocas de producción (otoño y primavera). En ambos experimentos, el diseño experimental fue en bloques completos al azar con parcelas divididas y 4 repeticiones.

Los datos fueron sometidos a análisis de varianza, utilizando la prueba de Tukey al 5\% para la diferenciación entre medias de tratamientos. El paquete estadístico utilizado fue INFOSTAT (Di Rienzo et al, 2014).

\section{RESULTADOS Y DISCUSIÓN}

\section{Cultivo Otoño-Invernal}

\section{Uso de lombricompuesto}

Durante el crecimiento del cultivo no se observaron diferencias significativas $(p>0,05)$ en el ensayo con aplicación de lombricompuesto entre los tratamientos, sin embargo a cosecha la interacción entre cultivar y dosis fue significativa $(p$ $<0,0001)$. 'Daguan' tuvo los valores de nitratos más bajos, independientemente de la fertilización. 'Brisa' tuvo concentraciones de nitratos más elevadas especialmente cuando fue abonada con lombricompuesto (Fig.1). Hubo una marcada tendencia decreciente de la concentración de nitratos desde los 37 días desde transplante a cosecha.

Los valores hallados concuerdan con Escobar-Gutiérrez et al. (2002) que informan que lechugas del tipo mantecosa tuvieron menores concentraciones de nitratos que otros tipos comerciales. Además, los valores de 'Brisa' concuerdan con otros autores (Byrne et al., 2001) que informaron que el contenido de nitratos en planta aumentó con la mayor disponibilidad de nitrógeno del suelo. Seginer (2003) encontró que cultivos de lechuga sometidos a estrés nitrogenado presentaron muy bajos contenidos de nitratos.

Tsai et al. (2005) determinaron que la absorción de nitrógeno por parte de los cultivos fue mayor cuando se incrementó la dosis de abono orgánico adicionado. A su vez, esto elevó el contenido de nitratos en los tejidos. De Martin y Restani (2003) informaron que hortalizas de hoja como endivias y lechugas de hojas crespas tuvieron contenidos de nitratos más altos cuando fueron cultivadas bajo un sistema orgánico comparado con un sistema convencional. Por el contrario, otros autores encontraron menor contenido de nitratos en hojas de lechuga con fertilizantes orgánicos frente a los de síntesis química que se utilizan en sistemas

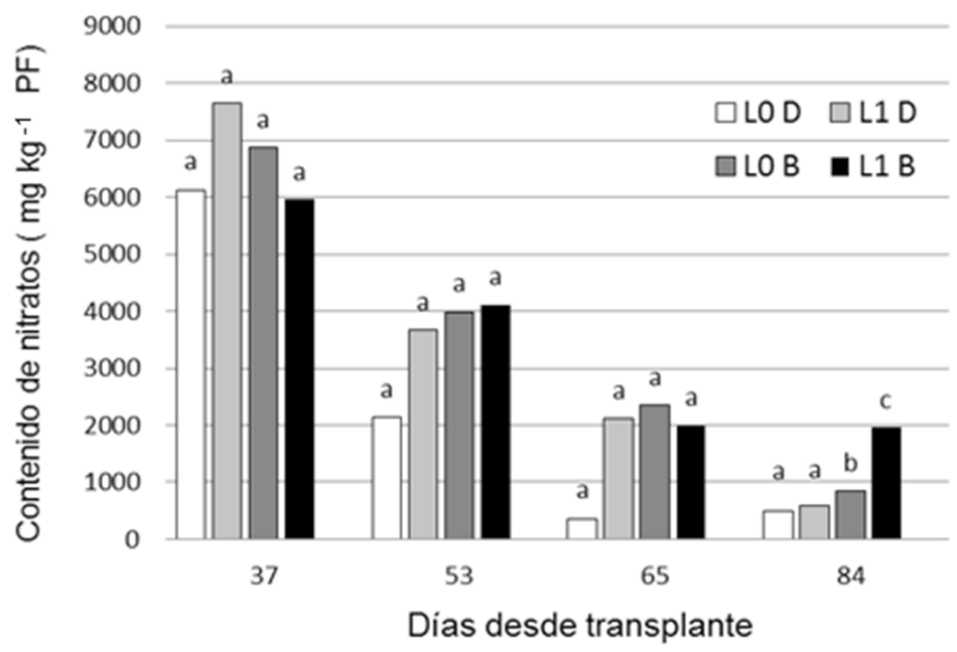

Fig. 1. Contenido de nitratos durante el crecimiento de lechuga cv. Daguan (D) y Brisa (B) en otoñoinvierno para el control (L0) y fertilización con lombricompuesto (L1). Diferentes letras muestran diferencias significativas entre tratamientos en cada fecha de muestreo $(\alpha=0,05)$.

Fig. 1. Nitrate content during lettuce growth cv. Daguan (D) and Brisa (B) in Autumn-Winter for control (L0) and fertilization with compost (L1). Different letters show significant differences between treatments in the same date $(\alpha=0.05)$. 
convencionales (Xu et al, 2003; Torregrosa et al, 2002).

Las diferencias encontradas por la aplicación del lombricompuesto fueron más evidentes en 'Daguan' que en 'Brisa' a lo largo del cultivo. Numerosos autores citan variaciones en el contenido de nitratos entre cultivares de lechuga (Amr y Hadidi, 2001; Escobar-Gutierrez et al., 2002; Siomos et al., 2002).

Se observó una tendencia general a la reducción en el contenido de nitratos a medida que avanza el desarrollo del cultivo en coincidencia con otros autores. Broadley et al. (2003) informaron que el contenido de nitrógeno orgánico y nitratos decrecen a medida que el peso fresco de la parte aérea de plantas de lechuga del cultivar Kennedy aumenta y se aproxima la fecha de cosecha. Seginer (2003) determinó que el contenido de nitratos en las plantas decreció con el tiempo mientras que el contenido de agua se incrementó entre la siembra y la cosecha.

La reducción media en el contenido de nitratos observada desde los 37 días desde transplante (DDT) hasta cosecha en 'Daguan' fue de 6551,3 a $534,2 \mathrm{mg} \mathrm{kg}^{-1} \mathrm{PF}$. Esto equivale a un $91,9 \%$. Entre los 60-65 DDT 'Daguan' inició la formación de la cabeza y desde ese momento hasta cosecha dicho cultivar experimentó una reducción en el contenido de nitratos de $56,8 \%$. Los elevados contenidos hallados en los estadios tempranos del cultivo en este ensayo de otoño-inverno serían concordantes con: a) la tendencia decreciente a lo largo del ciclo del cultivo hacia menores contenidos de nitratos, y b) los contenidos más elevados (más de 5000-6000 $\mathrm{mg} \mathrm{kg}{ }^{-1} \mathrm{PF}$ ) que se han obtenido a cosecha en otras experiencias. Al respecto, Byrne et al. (2001) observaron en algunos cultivos de lechuga crecidos durante el período otoño invernal, concentraciones superiores a $5000 \mathrm{mg} \mathrm{kg}^{-1} \mathrm{PF}$ a cosecha.

Los elevados contenidos de nitratos en las fases tempranas del cultivo pudieron estar asociados con el mantenimiento de la absorción de agua cuando el sistema radical aún estaba en desarrollo. Además de la función de inducir la síntesis de la nitrato reductasa, los nitratos, conjuntamente con la luz podrían actuar como una "señal" alterando la partición del flujo de carbono producido por la fotosíntesis hacia las hojas (Marschner, 2012) transformándolas en el destino principal.

Los valores hallados desde los 65 DDT hasta la cosecha no superaron el máximo nivel admitido por la Unión Europea (UE). Carrasco et al. (2006) también informaron que diferentes cultivares de lechuga cultivados durante el período invernal no superaron los límites establecidos por UE. Escalona et al. (2009) informaron que las fuentes de nitrógeno y dosis empleadas para lechugas cultivadas al aire libre tampoco sobrepasan los valores límite.

\section{Uso de fertilización convencional}

El contenido de nitratos presentó diferencias significativas a cosecha $(p<0,0001)$ debido a la interacción cultivar-dosis de urea. Durante el cultivo hubo una tendencia general en todos los tratamientos a la reducción del contenido de nitratos (Fig. 2).

A cosecha se encontraron valores más altos de nitratos en el cultivar Brisa a medida que aumentó la dosis de fertilizante aplicado. Los resultados coinciden con Sánchez (2010) que determinó un contenido de nitratos en lechuga de hojas crespas significativamente mayor con las dosis de $150 \mathrm{y}$ $200 \mathrm{~kg} \mathrm{~N} \mathrm{ha}^{-1}$.

En 'Daguan' se observó el mismo comportamiento sólo hasta una dosis de $75 \mathrm{~kg} \mathrm{~N} \mathrm{ha}^{-1}$ ya que con la mayor dosis la concentración observada fue menor. Es posible que la causa de este resultado sea un efecto de dilución debido al mayor peso fresco que tuvo 'Daguan' a cosecha en N2 respecto N1, si bien esa diferencia no fue estadísticamente significativa.

Weightman et al. (2006) determinaron que el sombreado incrementó significativamente el contenido de nitratos de siete cultivares de lechuga cuando se realizó entre 10 a 14 días previos a la cosecha. Además, en el campo constató el sombreado entre las hojas más externas como consecuencia del tamaño y morfología de la cultivar. Una posible explicación a los mayores contenidos de nitratos en este cultivar y a cosecha pudo deberse al efecto negativo del sombreado sobre la actividad de la nitrato reductasa en hojas externas.

A cosecha el orden de las cultivares según los niveles de nitratos en sus tejidos concuerdan con los de Escobar-Gutierrez et al. (2002) que informaron que lechugas del tipo mantecosa tuvieron menores concentraciones de nitratos que otros tipos comerciales.

A los 65 DDT el comportamiento de 'Brisa' fue similar al de la cosecha. Es decir, con mayores contenidos de nitratos a medida que se incrementó la dosis de fertilizante. 'Daguan' mostró otro patrón que sugiere una respuesta a la fertilización diferente a la observada en la cosecha. A los 65 DDT se observó una mínima diferencia significativa (DMS) tan grande como los valores medios medidos. Esto pudo haber sido provocado por la cercanía temporal de esta medición respecto a la fecha de fertilización (12 días). La urea se hidroliza muy rápidamente y genera una alta disponibilidad de nitrógeno para el cultivo. En condiciones ambientales de invierno (baja radiación y temperatura), el cultivo tiene más limitada la capacidad de reducir los nitratos que de absorberlos. Por lo tanto, se genera un desbalance que resulta en la acumulación de nitratos. 


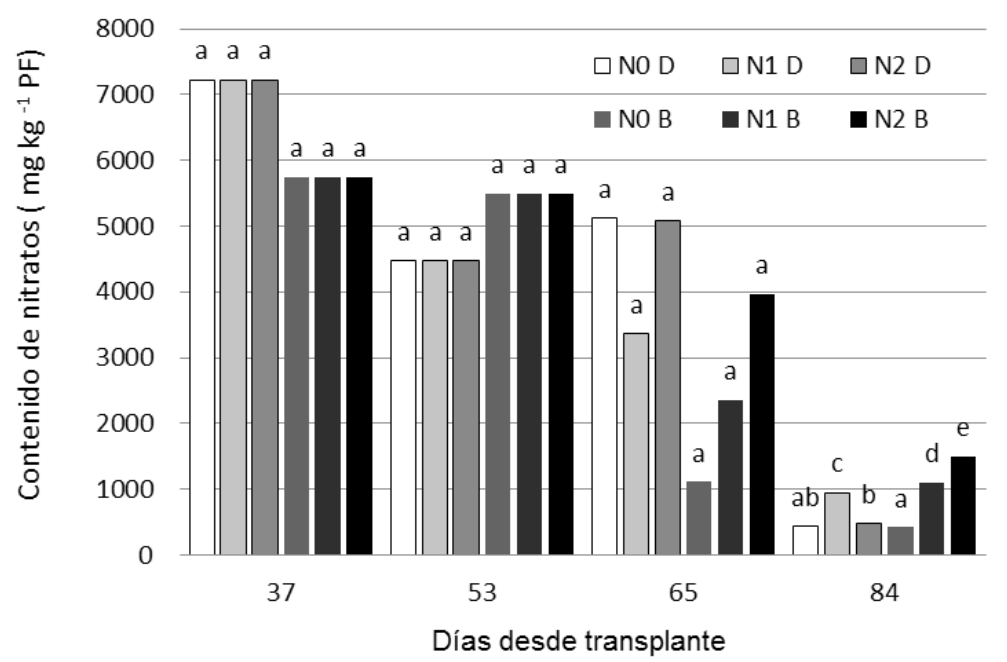

Fig. 2. Contenido de nitratos durante el crecimiento de cultivo de lechuga cv. Daguan (D) y Brisa (B) con distintas dosis de fertilizantes (N0 = Testigo sin fertilizar, N1 = $75 \mathrm{~kg} \mathrm{~N} \mathrm{ha}^{-1}, \mathrm{~N} 2=150 \mathrm{~kg} \mathrm{~N}$ $\left.\mathrm{ha}^{-1}\right)$. Letras distintas indican diferencias estadísticamente significativas según test de Tukey $(\alpha=0,05)$ para cada fecha de evaluación $(37,53,65$ y 84 días desde transplante).

Fig. 2. Nitrate content during lettuce crop growth for cv Daguan (D) and Brisa (B) and fertilization treatment (N0 = unfertilized, $\left.\mathrm{N} 1=75 \mathrm{~kg} \mathrm{~N} \mathrm{ha}^{-1}, \mathrm{~N} 2=150 \mathrm{~kg} \mathrm{~N} \mathrm{ha}^{-1}\right)$. Different letters show significant differences according to Tukey test $(\alpha=0.05)$ in each evaluation date $(37,53,65$ and 84 days after transplanting).

Independientemente que la fertilización se realizó de manera tal que la distribución de la urea fuese homogénea, pudo haber ocurrido que algunas plantas tuvieran mayor contacto con el fertilizante que otras. También, las lluvias pudieron eliminar parte del nitrógeno concentrándolo en algunos sitios por escurrimiento y posterior secado de la superficie del suelo. Esto significa que algunas plantas de manera individual pudieron estar sometidas a disponibilidades mayores que 75 y $150 \mathrm{~kg} \mathrm{~N} \mathrm{ha}^{-1}$ y otras a menores, de manera tal que la variabilidad en las observaciones pudo haberse visto incrementada.

$\mathrm{Al}$ igual que en el otro ensayo de otoño-invierno, se observó una tendencia general a la reducción en el contenido de nitratos a medida que avanzó el desarrollo del cultivo. La reducción media de nitratos en 'Daguan' durante todo el ciclo fue de 7224 a $617,6 \mathrm{mg} \mathrm{kg}^{-1} \mathrm{PF}$, es decir de $91,5 \%$, aunque $56,5 \%$ ocurrió en los últimos 19 días, período que se inició con la formación de las cabezas. Esto fue mayor a lo informado por Drews et al. (1996).

Los valores hallados hasta los 65 DDT superaron el máximo nivel permitido por la Unión Europea de $4500 \mathrm{mg} \mathrm{kg}^{-1} \mathrm{PF}$, en especial el cultivar 'Daguan'. Sin embargo, no es relevante para el consumidor ya que aún no ha alcanzado el estado de cosecha por tratarse de una lechuga de tipo mantecosa. Por el contrario, los valores a cosecha no superaron el máximo nivel permitido por la Unión Europea. Carrasco et al. (2006) también informaron que diferentes cultivares de lechuga cultivados durante el período invernal no superaron los límites establecidos por la Unión Europea.

\section{Cultivo Primaveral \\ Uso de lombricompuesto}

La concentración de nitratos fue significativamente mayor ( $p=0,0001)$ en 'Brisa' que en 'Daguan' al momento del transplante. A los 39 días no se encontraron diferencias significativas $(p>$ $0,05)$ en el ensayo con aplicación de lombricompuesto y a cosecha la interacción entre la dosis de fertilizante y el cultivar fue significativa $(p=$ 0,0003) de modo que L1 B fue el tratamiento con mayor contenido de nitratos seguido de LO B y por último L1 D (Fig. 3) En este caso, el suelo tiene un contenido de $0,26 \%$ de $\mathrm{N}$ total (Tabla 1 ), que aporta suficiente $\mathrm{N}$ por hectárea para lograr una adecuada nutrición nitrogenada del cultivo de lechuga (Havlin et al., 1999). Sin embargo, el abono orgánico adicionado elevó el contenido de nitratos en los tejidos a cosecha. Los valores de nitratos hallados durante el ciclo del cultivo no superaron el máximo nivel permitido por la Unión Europea.

En primavera la temperatura de suelo registró valores más elevados que en otoño y los fotoperíodos son mayores. Por ende, la contribución de 
las raíces a la reducción de los nitratos pudo ser mayor. Además, la función osmótica que tiene la acumulación de nitratos (Tei et al., 2000) pudo no ser tan importante en la primavera para el mantenimiento de las tasas de crecimiento. Sobre todo cuando el cultivo se encontraba en la primera fase de crecimiento. Esto estaría más relacionado a un cambio en el diferencial de potencial agua $(\Psi$ a) entre el suelo y la planta. Las mayores temperaturas del suelo en primavera implican una reducción de las fuerzas (adsorción, tensión superficial) que retienen el agua en el suelo con un consecuente incremento de su energía libre (Ratto, 1998; Villalobos et al., 2009).

\section{Uso de fertilización convencional}

A los 39 DDT no se observaron diferencias ( $p$ $=0,441)$ entre las cultivares en el caso del ensayo con aplicación de distintas dosis de urea (Fig. 4). A cosecha el tratamiento con mayor contenido de nitratos fue el cv. Brisa fertilizado con $150 \mathrm{~kg} \mathrm{~N}$ $\mathrm{ha}^{-1}(p<0,0001)$. Los resultados a cosecha muestran similitud con Byrne et al. (2001).

A cosecha el orden de las cultivares según los niveles de nitratos en sus tejidos concuerdan sólo de manera parcial con Escobar-Gutiérrez et al. (2002), esto es, al transplante y siendo sólo aplicable en relación a N2 B en la cosecha. Este resultado concuerda con Sánchez (2010) que trabajando con lechuga de hojas crespas determinaron un contenido de nitratos significativamente mayor cuando las dosis de nitrógeno fueron de 150 y 200 $\mathrm{kg} \mathrm{N} \mathrm{ha-1}$. El cultivo en condiciones ambientales de primavera (radiación y temperatura en aumento) incrementó tanto su capacidad de reducir los nitratos como de absorberlos.

Se observó una tendencia general a la reducción en el contenido medio de nitratos desde el momento de la fertilización. Algo similar encontraron otros autores. La reducción media en el contenido de nitratos entre los 39 y 60 DDT observada en 'Daguan' fue de 935,3 a 412,5 mg kg ${ }^{-1} \mathrm{PF}$, es decir $55,9 \%$ en un período de 21 días. El inicio de la formación de la cabeza ocurrió entre los 37 y 45 DDT, por lo tanto, esta observación es mayor a la de Drews et al. (1996).

Por un lado, en primavera la temperatura de suelo registró valores más elevados que en otoño, además de mayores fotoperíodos. Por ende, la contribución de las raíces a la reducción de los nitratos pudo ser más elevada y la acumulación de los mismos en el vástago tuvo otro factor limitante. Por otro lado, la función osmótica que tiene la acumulación de nitratos (Tei et al., 2000) pudo no ser tan importante en la primavera para el mantenimiento de altas tasas de crecimiento cuando el cultivo se encontraba en la fase de crecimiento exponencial. Esto estaría relacionado a un cambio

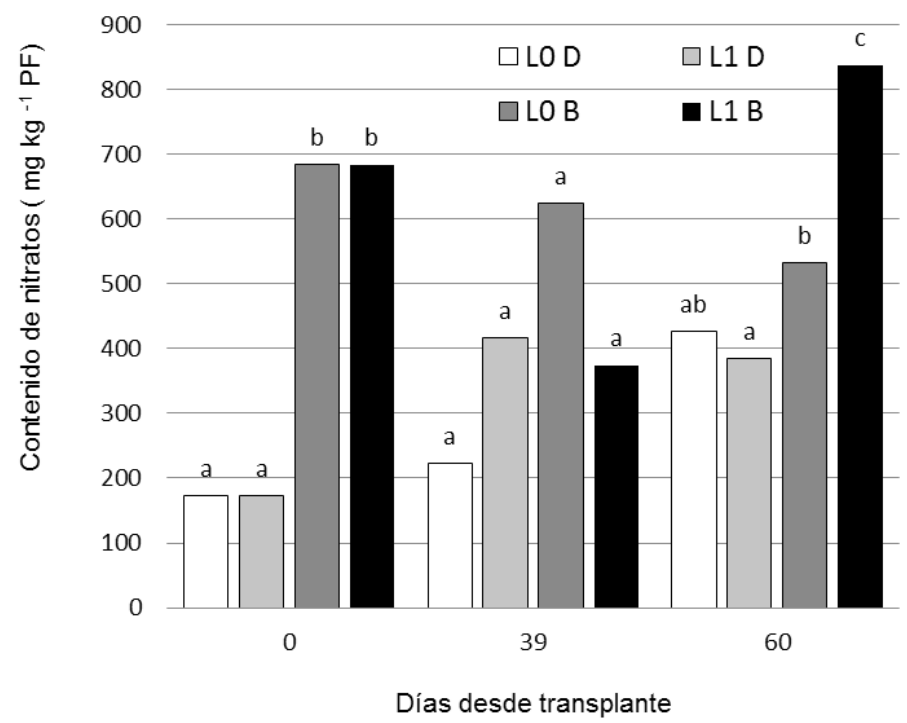

Fig. 3. Contenido de nitratos durante el crecimiento de cultivo de lechuga cv. Daguan (D) y Brisa (B) para el tratamiento control (L0) y con lombricompuesto (L1) en primavera. Letras distintas indican diferencias estadísticamente significativas $(\alpha=0,05)$ entre material genético y tratamiento de fertilización a cosecha y a los 39 y 60 días desde transplante.

Fig. 3. Nitrate content during lettuce crop growth for cv. Daguan (D) and Brisa (B) and fertilization treatment $(\mathrm{L} 0=$ unfertilized, $\mathrm{L} 1=$ vermicompost $)$ in spring. Different letters show significant differences according to Tukey test $(\alpha=0.05)$ between genetic material and fertilization treatment in each date at harvest, 39 and 60 days after transplanting. 


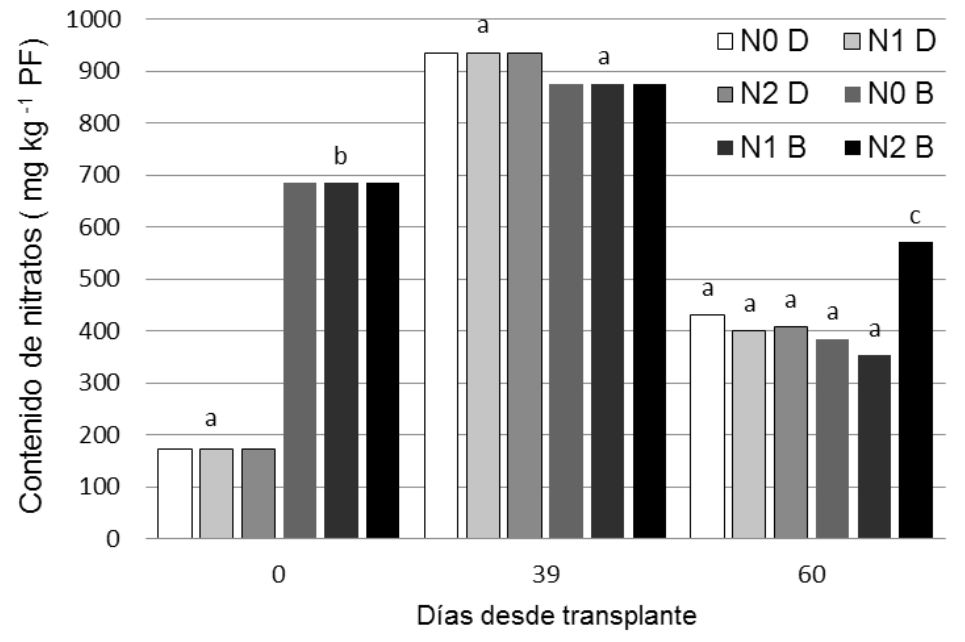

Fig. 4. Contenido de nitratos ( $\left.\mathrm{mg} \mathrm{kg}^{-1} \mathrm{PF}\right)$ durante el crecimiento de cultivo de lechuga cv. Daguan (D) y Brisa (B) en primavera según tratamiento de fertilización (N0 = Testigo sin fertilizar, $\left.\mathrm{N} 1=75 \mathrm{~kg} \mathrm{~N} \mathrm{ha}^{-1}, \mathrm{~N} 2=150 \mathrm{~kg} \mathrm{~N} \mathrm{ha}^{-1}\right)$. Letras distintas indican diferencias estadísticamente significativas según test de Tukey $(\alpha=0,05)$ para cada fecha de evaluación $(0,39$ y 60 días desde transplante).

Fig. 4. Nitrate content ( $\left.\mathrm{mg} \mathrm{kg}^{-1} \mathrm{FW}\right)$ during lettuce crop growth for cv. Daguan (D) and Brisa (B) in spring for fertilization treatment (N0 = unfertilized, $\mathrm{N} 1=75 \mathrm{~kg} \mathrm{~N} \mathrm{ha}^{-1}, \mathrm{~N} 2=150 \mathrm{~kg} \mathrm{~N} \mathrm{ha}^{-}$ $\left.{ }^{1}\right)$. Different letters show significant differences according to Tukey test $(\alpha=0.05)$ in each evaluation date $(0,39$ and 60 days after transplanting).

en el diferencial de potencial agua entre el suelo y la planta.

\section{CONCLUSIONES}

Los resultados indican una mayor concentración de nitratos en cultivos de invierno respecto de primaverales, que se manifestó en ambos cultivares evaluados, con mayor efecto en el cultivar Brisa. Por lo tanto, se observó que el contenido de nitratos depende del cultivar. Para estudiar el efecto de fertilizantes tanto químicos como orgánicos sobre otros cultivares deben realizarse nuevos estudios ya que el factor genético es uno de los más influyentes en el contenido de nitratos.

En ningún caso los valores de nitratos en lechuga a cosecha superaron los umbrales máximos tolerados por las normativas de la Comunidad Europea por lo cual puede afirmarse que son alimentos seguros.

\section{LITERATURA CITADA}

Albornoz, L. 2000. Contenido de nitratos en deshidratado de espinacas. 82 p. Tesis para optar por el título de la Licenciatura en Bromatología. Facultad de Ciencias Agrarias, Universidad Nacional de Cuyo, Mendoza, Argentina.
Amr, A., and N. Hadidi. 2001. Effect of cultivar and harvest date on nitrate $\left(\mathrm{NO}_{3}\right)$ and nitrite $\left(\mathrm{NO}_{2}\right)$ content of selected vegetables grown under open field and greenhouse conditions in Jordan. Journal of Food Composition and Analysis 14(1):59-67.

Azcon-Bieto, J., y M. Talon. 2000. Fundamentos de fisiología vegetal. 522 p. Ed. Mc Graw Hill/ Interamericana de España, Madrid, España.

Bahadoran, Z., P. Mirmiran, S. Jeddi, F. Azizi, A. Ghasemi, and F. Farzad Hadaegh. 2016. Nitrate and nitrite content of vegetables, fruits, grains, legumes, dairy products, meats and processed meats. Journal of Food Composition and Analysis 51:93-105.

Broadley, M.R., I. Seginer, A. Burns., A.J. Escobar-Gutierrez., I.J. Burns, and P.J.White. 2003. The nitrogen and nitrate economy of butterhead lettuce (Lactuca sativa var. capitata L.). Journal of Experimental Botany 54(390):2081-2090.

Burns, I.G., A. Lee, and A.J. Escobar Gutierrez. 2004. Nitrate accumulation in protected lettuce. Acta Horticulturae 633:271-278.

Byrne, C., M.J. Maher, M.J. Hennerty, M.J. Mahon, and P.A. Walshe. 2001. Reducing the nitrate content of protected lettuce. Kinsealy Research Centre, Dublin, Ireland. 
Carrasco, G., J. Tapia, y M. Urrestarazu. 2006. Contenido de nitratos en lechugas cultivadas en sistemas hidropónicos. IDESIA (Chile) 24(1):25-30

Cataldo, D.A., M. Haroon, L.E. Schrader, and V.L. Youngs. 1975. Rapid colorimetric determination of nitrate in plant tissue by nitration of salicylic acid. Common. Soil Science and Plant Analysis 6(1):71-80.

Chiesa, A. 2010. Factores precosecha y postcosecha que inciden en la calidad de la lechuga. Horticultura Argentina 29(68):28-32.

Chowdhury, A., and A. Das. 2015. Nitrate accumulation and vegetable quality. International Journal of Science and Research 4(12):1668-1672.

Comunidad Europea. 2006. Reglamento No. 1881/2006. Contenido máximo de determinados contaminantes en los alimento. Comisión de las Comunidades Europeas (CE). Diario Oficial de la Unión Europea. 19 de diciembre de 2006. Bruselas, Bélgica.

De Martin, S., and P. Restani. 2003. Determination of nitrates by a novel ion chromatographic method: occurrence in leafy vegetables (organic and conventional) and exposure assessment for Italian consumers. Food Additives and Contaminants 20(9):787-792.

de Grazia, J., P.A. Tittonell, Á. Chiesa. 2001. Efecto de la época de siembra, radiación y nutrición nitrogenada sobre el patrón de crecimiento y el rendimiento del cultivo de lechuga (Lactuca sativa L.). Investigación Agraria: Producción y Protección Vegetal 16(3):355-365.

Di Rienzo J.A., F. Casanoves, M.G. Balzarini, L. Gonzalez, M. Tablada, y V.W. Robledo. 2014. InfoStat versión 2014. Grupo InfoStat, FCA, Universidad Nacional de Córdoba, Argentina. Disponible http://www.infostat.com.ar (Consulta 07 septiembre 2015).

Drews, M., I. Schonhof, and A. Krumbein. 1996. Influence of growth season on the content of nitrate, vitamin $\mathrm{C}$, b-carotene, and sugar of head lettuce under greenhouse conditions. Gartenbauwissenschaft 60:180-187.

Escalona A., M. Santana, I. Acevedo, V. Rodríguez, y L.M. Marcó. 2009. Efecto de las fuentes nitrogenadas sobre el contenido de nitratos y lecturas "spad" en el cultivo de lechuga. Agronomía Tropical 59(1):99-105.

Escobar Gutierrez, A.J., I.G. Burns., A. Lee, and R.N. Edmonson. 2002. Screening lettuce cultivars for low nitrate content during summer and winter production. Journal of Horticultural Science and Biotechnology 77(2):232-237.
Fernández Lozano, J. 2012. La producción de hortalizas en Argentina. 29 p. Gerencia de Calidad y Tecnología. Mercado Central de Buenos Aires, Buenos Aires, Argentina.

Filippini, M.F. 2006. Contenido en productos frutihorticolas, 2do Foro FANUS, Nitratos y Nitritos en la Alimentación Humana. 22 de Septiembre. Bolsa de Cereales, Buenos Aires, Argentina.

Harrison, N. 2001. Inorganic contaminants in food. p. 163-165. In Watson, D.H. (ed.) Food chemical safety. Contaminants. Woodhead Publishing, Cambridge, England.

Havlin, J.L., J.D. Beaton, S.L. Tisdale, and W.L. Nelson. 1999. Soil Fertility and Fertilizers. 499 p. 6th ed. Prentice Hall, Upper Saddle River, New Jersey, USA.

Hord, N.G., Y. Tang, and N.S. Bryan. 2009. Food sources of nitrates and nitrites: the physiologic context for potential health benefits. American Journal of Clinical Nutrition 90:1-10.

Konstantopoulou, E., G. Kapotis, G. Salachas, S.A. Petropoulos, I.C. Karapanos, and H.C. Passam. 2010. Nutritional quality of greenhouse lettuce at harvest and after storage in relation to $\mathrm{N}$ application and cultivation season. Scientia Horticulturae 125:93.e1-93.e5.

Koudela, M., and K. Petøíková. 2008. Nutrients content and yield in selected cultivars of leaf lettuce (Lactuca sativa L. var. crispa). Horticultural Science (Prague) 35(3):99-106.

Marschner, P. 2012. Mineral nutrition of higher plants. 651 p. 3er ed. Academic Press/Elsevier, London, England.

Pavlou, G.C, C.D. Ehaliotis, and V.A. Kavvadias. 2007. Effect of organic and inorganic fertilizers applied during successive crop seasons on growth and nitrate accumulation in lettuce. Scientia Horticulturae 111:319-325.

Ratto, S. 1998. Agua del suelo. p. 201-233. En Principios de Edafología con énfasis en suelos argentinos. Editorial Gráfica Editora SRL, Buenos Aires, Argentina.

Sánchez, T.M. 2010. Evaluación de la calidad de lechuga (Lactuca sativa L.) respecto a su contenido de nitratos y materia seca. Revista de la Facultad de Agronomía de la Universidad de La Pampa 21:29-36

Secretaría de Agricultura, Ganadería, Pesca y Alimentos. 2007. Resolución 256/2007. Rotulación de los alimentos envasados que contengan vegetales y una cantidad de nitratos superior a la indicada. Secretaría de Agricultura, Ganadería, Pesca y Alimentos (SAGPyA), Buenos Aires, Argentina. 
Seginer, I. 2003. A dynamic model for nitrogen-stressed lettuce. Annals of Botany 91:623-635.

Siomos, A.S., P.P. Papadopoulou, C.C. Dogras, E. Vasiliadis, A. Dosas, and N. Georgiou. 2002. Lettuce composition as affected by genotype and leaf position. Acta Horticulturae 579:635-639.

Tei, F., P. Benincasa, and M. Guiducci. 2003. Critical nitrogen concentration in lettuce. Acta Horticulturae 627:187-194.

Tei, F., P. Benincasa, and M. Guiducci. 2000. Effect of nitrogen availability on growth and nitrogen uptake in lettuce. Acta Horticulturae 533:385-392.

Torregrosa, S., M.D. Raigón, A. Dominguez, y J.M Carot. 2002. Relació sol-planta en sistemas de producción ecologic i convencional en funció del tipus de reg en conreu de l'encisam romà. 159 p. Trabajo Final de Carrera. Escuela Técnica Superior del Medio Rural y Enología. Universidad Politécnica de Valencia, España.

Tsai, Y.H., H.M. Hsu, and R.S. Chung. 2005. The effect of application of different rates of organic fertilizer on the soil properties and nitrogen uptake of vegetables planted in plastic house. Journal of the Agricultural Association of China 6(3):229-244.
Weightman, R.M., C. Dyer, J. Buxton, and D.S. Farrington. 2006. Effects of light level, time of harvest and position within field on the variability of tissue nitrate concentration in commercial crops of lettuce (Lactuca sativa) and endive (Cichorium endivia). Food Additives and Contaminants 23(5):462-469.

WHO. 1995. Nitrite and nitrate. In Toxicological evaluation of certain food additives and contaminants in food. Prepared by the 44th report of the joint $\mathrm{FAO} / \mathrm{WHO}$ experts committee on food additives. Food Additives Series 35:269-360.

Villalobos, F.J., I. Mateos, F. Orgaz, y E. Fereres. 2009. Fitotecnia: Bases y tecnologías de la producción agrícola. 496 p. Ediciones Mundiprensa, Madrid, España.

Xu, H.L., Wang, R., Xu, R.Y., Mridha, M.A.U. and S. Goyal. 2003. Yield and quality of leafy vegetables grown with organic fertilizations. Acta Horticulturae 627:25-33. 